Bull. Austral. Math. Soc.

VOL. $39(1989) \quad[21-24]$

\title{
HYPERTYPES OF TORSION-FREE ABELIAN GROUPS OF FINITE RANK
}

\author{
H.P. Goeters, C. Vinsonhaler and W. Wickless
}

\begin{abstract}
Let $G$ be a torsion-free abelian group of finite rank $n$ and let $F$ be a full free subgroup of $G$. Then $G / F$ is isomorphic to $T_{1} \oplus \ldots \oplus T_{n}$, where $T_{1} \subseteq T_{2} \subseteq \ldots \subseteq T_{n} \subseteq \mathbb{Q} / \mathbb{Z}$. It is well known that type $T_{1}=$ inner type $G$ and type $T_{n}=$ outer type $G$. In this note we give two characterisations of type $T_{i}$ for $1<i<n$.
\end{abstract}

In 1963 Fuchs [2] introduced the notions of the inner type (IT) and outer type (OT) of a torsion-free abelian group $G$ of finite rank $n$. He showed that if $F$ is a full free subgroup of $G$ and $G / F=T_{1} \oplus \ldots \oplus T_{n}$ where the $T_{i}$ are subgroups of $\mathrm{Q} / \mathbb{Z}$ with $T_{1} \subseteq T_{2} \subseteq \ldots \subseteq T_{n}$, then $I T(G)=\operatorname{type}\left(T_{1}\right)$ and $O T(G)=\operatorname{type}\left(T_{n}\right)$. In this note we generalise the result of Warfield by characterising type $\left(T_{i}\right)$, the $i$ th hypertype of $G$, for $1 \leqslant i \leqslant n$.

Fundamental references are $[\mathbf{1}, \mathbf{2}, 4]$ and [5]. In particular, the reader is assumed to be familiar with the basic properties. of height and type in torsion-free abelian groups, and with the notions of inner and outer type. We also assume familiarity with quasiisomorphism concepts.

If $A$ and $B$ are groups we write $A \leqslant B$ to denote that $A$ is isomorphic to a subgroup of $B$. For an integral prime $p, A_{p}$ clenotes the usual localisation of $A$ at $p$. If $G$ is a torsion-free abelian group and $S$ is a subset of $G$, then $\langle S\rangle_{*}$ is the pure subgroup generated by $S$. If $x \in G$, then $h_{p}^{G}(x)$ is the $p$-height of $x$ computed in $G$. If $G$ has rank $n$ and $0 \leqslant i \leqslant n$, we define $P_{i}(G)=\{X \mid X$ is a pure subgroup of $G$ of rank $i\}$.

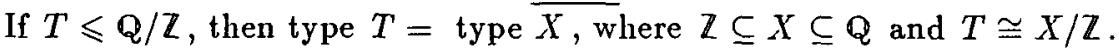

If $T \leqslant(\mathbb{Q} / \mathbb{Z})^{n}$, then it is easy to see that $T$ can be written as $T=\oplus_{i=1}^{n} T_{i}$ where $T_{1} \leqslant T_{2} \leqslant \ldots \leqslant T_{n} \leqslant Q / Z$. We say such a direct sum is a standard decomposition. Thus, with each $T \leqslant(\mathbb{Q} / Z)^{n}$ we can associate a set of types, typeset $T=$ type $T_{1}, \ldots$, type $\left.T_{n}\right\}$, where $T=\oplus T_{i}$ is a standard decomposition. It is easy to check that typeset $T$ is a complete set of quasi-isomorphism invariants for subgroups $T$ of $(\mathbb{Q} / \mathbb{Z})^{n}$.

Let $G$ be a torsion-free abelian group of rank $n$ - henceforth simply called a "group". The Richman type of $G, R T(G)$, is the quasi-isomorphism class of the torsion

Received 8 March 1988

The authors are grateful to the referee of a previous version of this paper. His suggestions considerably shortened the proofs of Theorems 1 and 2 .

Copyright Clearance Centre, Inc. Serial-fee code: $0004-9729 / 89 \quad \$ A 2.00+0.00$. 
group $G / F$, where $F$ is any full free subgroup of $G$. The quasi-isomorphism class of $G / F$, denoted $[G / F]$, is independent of the choice of $F$ so that $R T(G)$ is an invariant of $G([3])$. Furthermore, since $G / F \leqslant(Q / Z)^{n}$, our earlier remarks imply that $R T(G)$ is determined by the set of types typeset $(G / F)$. If $G / F=\oplus_{i=1}^{n} T_{i}$ is a standard decomposition, we call $H T_{i}(G)=$ type $T_{i}$ the ith hypertype of $G$. As mentioned above, $H T_{1}(G)=I T(G)$ and $H T_{n}(G)=O T(G)$.

The properties of $\mathrm{HT}_{2}(G)$ will be investigated in a forthcoming paper [3]. Our main result here, which leads to the desired characterisations of hypertypes, displays the relationship between successive hypertypes.

TheOREM 1. Let $G$ be a group of rank $n>1$. Then for $2 \leqslant i \leqslant n$,

$$
H T_{i}(G)=\sup \left\{H T_{i-1}(G / X) \mid X \in P_{1}(G)\right\}
$$

Proof: Let $\left\{x_{1}, \ldots, x_{n}\right\}$ be a maximal rationally independent subset (hereafter, basis) of $G$, and $F=\oplus_{i=1}^{n} Z x_{i}$, a full free subgroup of $G$. Let $G / F=\oplus_{i=1}^{n} T_{i}$ be a standard decomposition of $G / F$. For each $X \in P_{1}(G),(F+X) / X$ is a full free subgroup of $G / X$. Write $(G / X) /((F+X) / X)=S_{1} \oplus \ldots \oplus S_{n-1}$ as a standard decomposition. Since $\oplus_{i=1}^{n-1} S_{i}$ is a homomorphic image of $\oplus_{i=1}^{n} T_{i}$ it follows that $\left|\left(T_{i}\right)_{p}\right| \geqslant\left|\left(S_{i-1}\right)_{p}\right|$ for all primes $p$ and $2 \leqslant i \leqslant n$. Since $H T_{i}(G)=$ type $T_{i}$ and $H T_{i-1}(G / X)=$ type $S_{i-1}$ we have $H T_{i}(G) \geqslant H T_{i-1}(G / X)$ for all $X \in P_{1}(G)$.

For a fixed prime $p$, choose an element $x_{i}$ of minimal $p$-height anong the basis elements, and let $X(p)=\left\langle x_{i}\right\rangle_{*}$. Note that if $e_{p}=p$-height $\left(x_{i}\right)=\min \left\{p\right.$ height $\left(x_{j}\right) \mid 1 \leqslant$ $j \leqslant n\}$, then $T_{1}=\oplus_{p} Z\left(p^{e_{p}}\right)$. Furthermore, for each $p$, the minimality of $e_{p}$ implies that $(X(p)+F)_{p} / F_{p}$ is a pure subgroup of $G_{p} / F_{p}$ and hence, a summand. Therefore, $G_{p} /(X(p)+F)_{p} \simeq\left(T_{2} \oplus \ldots \oplus T_{n}\right)_{p}$, so that $H T_{i}(G) \leq \sup \left\{H T_{i_{1}}\left(G /\left\langle x_{j}\right\rangle *\right) \mid 1 \leqslant j \leqslant\right.$ $n\}$. Thus, $H T_{i}(G) \leqslant \sup \left\{H T_{i-1}(G / X) \mid X \in P_{1}(G)\right\}$ and the theorem follows.

Remark. Using induction, we may extend the results in the proof of Theorem 1 to show that for each prime $p$, and $1 \leqslant i \leqslant n$, there is a subset $I$ of $\{1,2, \ldots, n\}$ of cardinality $i$, such that $Y=Y_{I} \equiv\left\langle X_{j} \mid j \in I\right\rangle_{*}$ satisfies $Y_{p} /(Y \cap F)_{p} \simeq\left(T_{1} \oplus \ldots \oplus T_{i}\right)_{p}$ and $G_{p} /(Y+F)_{p} \simeq\left(T_{i+1} \oplus \ldots \oplus T_{n}\right)_{p}$.

Corollary 1. Suppose there exists $x \in G$ such that type $x=I T(G)$. Then, if $X=\langle x\rangle_{*}, R T(G)=\left[\oplus_{i=1}^{n} T_{i}\right]$ implies that $R T(G / X)=\left[\oplus_{i=2}^{n} T_{i}\right]$.

Proof: Choose a basis $\left\{x=x_{1}, \ldots, x_{n}\right\}$ of $G$ such that $h_{p}^{G}(x) \leqslant h_{p}^{G}\left(x_{i}\right)$ for all $p$ and $2 \leqslant i \leqslant n$. Then in the notation of Theorem $1, X(p)=\langle x\rangle_{*}$ for all $p$, and the argument in the second half of the proof of Theorem 1 shows that $\left(T_{i}\right)_{p} \cong\left(S_{i-1}\right)_{p}$ for all primes $p$ and $2 \leqslant i \leqslant n$, where $G /(X+F)=S_{1} \oplus \ldots \oplus S_{n}$ is a standard decomposition. Thus $R T(G / X)=\left[\oplus_{i=1}^{n-1} S_{i}\right]=\left[\oplus_{i=2}^{n} T_{i}\right]$. 
Note that the hypothesis of Corollary 1 holds if $G$ is homogeneous. This corollary is proved for homogeneous groups in [3] using different techniques.

Corollary 2. Let $G$ be a group of rank $n$. Then, for $1 \leqslant i \leqslant n, H T_{i}(G)=$ $\sup \left\{I T(G / X) \mid X \in P_{i-1}(G)\right\}$.

Proof: The proof is by induction on $i$. For $i=1, H T_{1}(G)=I T(G)=$ $I T(G /(0))$, and the result is true. Assume $i>1$ and that the result holds for $i-1$. By Theorem 1 and the induction hypothesis, $H T_{i}(G)=\sup \left\{H T_{i-1}(G / X) \mid X \in\right.$ $\left.P_{1}(G)\right\}=\sup \left\{\sup \left\{I T\left((G / X) / Y^{\prime}\right) \mid Y^{\prime} \in P_{i-2}(G / X)\right\} \mid X \in P_{1}(G)\right\}$. However, each $Y^{\prime} \in P_{i-2}(G / X)$ is of the form $Y^{\prime \prime}=Y / X$ for a unique $Y \in P_{i-1}(G)$. Thus, $H T_{i}(G)=\sup \left\{\sup \left\{I T(G / Y) \mid X \subset Y \in P_{i-1}(G)\right\} \mid X \in P_{1}(G)\right\}=\sup \{I T(G / Y) \mid$ $\left.Y \in P_{i-1}(G)\right\}$, as required.

Note that for $i=n$ we have $O T(G)=H T_{n}(G)=\sup \{r T(G / X) \mid X \in$ $\left.P_{n-1}(G)\right\}=\sup \left\{\operatorname{type}(G / X) \mid X \in P_{n-1}(G)\right\}$, the standard definition of the outer type of $G$.

Arguing as in the proof of Corollary 2, it is easy to show:

COROLlary 3. Let $G$ be a group of rank $n$ and $1 \leqslant j<i \leqslant n$. Then $H T_{i}(G)=$ $\sup \left\{H T_{i-j}(G / X) \mid X \in P_{j}(G)\right\}$.

If $\tau$ is a type, a group $G$ is called a hyper- $\tau$ group if every proper homomorphic image of $G$ is $\tau$-homogeneous completely decomposable. These groups are investigated in $[3]$. One of the results in that paper may be generalised as follows (see [3], Theorem $3.1)$.

Corollary 4. Let $G$ be a hyper- $\tau$ group. Then $R T(G)=\left[S \oplus T^{n-1}\right]$, where type $S=I T(G)$ and type $T=\tau$.

Proof: If $G$ is a hyper- $\tau$ group then, clearly, $H T_{n}(G)=O T(G)=\tau$. But by Theorem 1, $H T_{2}(G)=\sup \left\{I T(G / X) \mid X \in P_{1}(G)\right\}=\tau$. Thus, $H T_{i}(G)=\tau$ for $i=2, \ldots, n$ and the result follows.

TheOREM 2. Let $G$ be a torsion-free group of rank $n$. Then for $1 \leqslant i \leqslant n$, $H T_{i}(G)=\inf \left\{O T(X) \mid X \in P_{i}(G)\right\}$.

Proof: Fix $Y$ in $P_{i}(G)$. If $F$ is a full free subgroup of $G$, then $F \cap Y$ and $(F+Y) / Y$ are full free subgroups of $Y$ and $G / Y$, respectively. Write $G / F=T_{1} \oplus$ $\ldots \oplus T_{n}, Y /(F \cap Y)=U_{1} \oplus \ldots \oplus U_{i}$ and $(G / Y) /((F+Y) / Y)=S_{1} \oplus \ldots \oplus S_{n-i}$ as standard decompositions. Then there is an exact sequence,

$$
0 \rightarrow \oplus_{j=1}^{i} U_{j} \rightarrow \oplus_{j=1}^{n} T_{j} \rightarrow \oplus_{j=1}^{n-i} S_{j} \rightarrow 0
$$


It is routine to show that type $U_{j} \geqslant$ type $T_{j}$ for $1 \leqslant j \leqslant i$. Since $O T(Y)=$ type $U_{i}$ and $H T_{i}(G)=$ type $T_{i}$, we have shown $O T(Y) \geqslant H T_{i}(G)$ for all $Y \in P_{i}(G)$. However, by the remark following the proof of Theorem 1, for each prime $p$, there is a subset $I$ of $\{1,2, \ldots, n\}$ of cardinality $i$ such that $Y=Y_{I}=\left\langle x_{j} \mid j \in I\right\rangle_{*} \in P_{i}(G)$ satisfies $Y_{p} /(Y \cap F)_{p} \simeq\left(T_{1} \oplus \ldots \oplus T_{i}\right)_{p}$. Thus, $H T_{i}(G)=$ type $T_{i} \leq \inf \left\{O T\left(Y_{I}\right) \mid\right.$ $I$ is a subset of $\{1, \ldots, n\}$ of cardinality $i\}$, and the proof is complete.

\section{References}

[1] D. Arnold, Finite Rank Torsion Free Abelian Groups and Rings: Lecture Notes in Math. 931 (Springer-Verlag, Berlin, Heidelberg, New York, 1982).

[2] L. Fuchs, 'Recent results and problems on Abelian groups', in Topics in Abelian Groups, pp. 9-40 (Scott and Foresman, Chicago, Illinois, 1963).

[3] H.P. Goeters and W. Wickless, 'Hyper- $\tau$ groups', Comm. Algebra (to appear).

[4] F. Riclıman, 'A class of rank-2 torsion free groups', Studies on Abelian Groups (B. Charles, Dunod, 1968), 327-333.

[5] R. Warfield, 'Homomorphisins and duality for torsion free groups', Math. Z. 107 (1968), 189-200.

H.P. Goeters,

Mathematics ACA,

Auburn University,

Auburn, AL 36849

United States of America

W. Wickless,

Department of Mathematics

University of Connecticut

196 Auditorium Rd

Storrs, CT. 06268

United States of America
C. Vinsonhaler,

Department of Mathematics

University of Connecticut

196 Auditorium Rd

Storrs, CT. 06268

United States of America 